\title{
$q$-Analogues and properties of the Laplace-type integral operator in the quantum calculus theory
}

\section{Shrideh Khalaf Al-Omari ${ }^{1 *}$}

"Correspondence:

shridehalomari@bau.edu.jo

${ }^{1}$ Department of Physics and Basic

Sciences, Faculty of Engineering

Technology, Al-Balqa Applied

University, 11134 Amman, Jordan

\begin{abstract}
In this paper, we discuss the q-Laplace-type integral operator on certain class of special functions. We propose $q$-analogues and obtain results involving polynomials of even orders and functions of $q$-trigonometric types. Moreover, we establish results related to $q$-hyperbolic functions and certain $q$-differential operators. Relying on the given $q$-differentiation formulas, we finally derive the $n$th derivative of the $q$-Laplace-type integral and attain formulas including $q$-convolution products.
\end{abstract}

MSC: Primary 54C40; 14E20; secondary 46E25; 20C20

Keywords: $q$-Laplace transform; $q$-Natural transform; q-Bessel function; Fox's $q$-H-functions

\section{Introduction}

The subject of quantum calculus is known as the calculus without limits; it has earned an eminent reputation and publicity due to its vast applications in mathematics, statistics and physics. Much of the theory of the quantum calculus relies on the Jackson $q$ derivatives and $q$-integrals, which replace the classical derivative by a difference operator, which allows one to deal with sets of non-differentiable functions. Recently, this area has been stimulated to grow rapidly by many researchers and a variety of new results can be found in Refs. [1-16] and the references cited therein. By fixing a real number $q$ such that $0<q<1$, the $q$-derivative of a differentiable function $\vartheta$ is defined by [1]

$$
D_{q} \vartheta(x)=\frac{\vartheta(x)-\vartheta(q x)}{(1-q) x} \quad(x \neq 0) .
$$

The $q$-integrals from 0 to $y$ and from 0 to $\infty$ are, respectively, defined by [1]

$$
\int_{0}^{y} \vartheta(x) d_{q} x=(1-q) y \sum_{n=0}^{\infty} \vartheta\left(y q^{n}\right) q^{n}
$$

(c) The Author(s) 2020. This article is licensed under a Creative Commons Attribution 4.0 International License, which permits use, sharing, adaptation, distribution and reproduction in any medium or format, as long as you give appropriate credit to the original author(s) and the source, provide a link to the Creative Commons licence, and indicate if changes were made. The images or other third party material in this article are included in the article's Creative Commons licence, unless indicated otherwise in a credit line to the material. If material is not included in the article's Creative Commons licence and your intended use is not permitted by statutory regulation or exceeds the permitted use, you will need to obtain permission directly from the copyright holder. To view a copy of this licence, visit http://creativecommons.org/licenses/by/4.0/. 
and

$$
\int_{0}^{\infty} \vartheta(x) d_{q} x=(1-q) \sum_{n=-\infty}^{\infty} \vartheta\left(q^{n}\right) q^{n}
$$

provided their respective series converge absolutely. The $q$-integration by parts is given by [16]

$$
\int_{a}^{b} g(x) D_{q} \vartheta(x) d_{q} x=\vartheta(b) g(b)-\vartheta(a) g(a)-\int_{a}^{b} \vartheta(q x) D_{q} g(x) d_{q} x .
$$

In the literature, there have been found two types of $q$-analogues of the exponential function introduced as [16-18]

$$
E_{q}(x)=\sum_{n=0}^{\infty} q^{\frac{n(n-1)}{2}} \frac{x^{n}}{[n]_{q} !} \quad(x \in \mathbb{C})
$$

and

$$
e_{q}(x)=\sum_{n=0}^{\infty} \frac{x^{n}}{[n]_{q} !} \quad\left(|x|<|1-q|^{-1}\right)
$$

where $[n]_{q}=q^{n-1}+\cdots+q+1$ and $[n]_{q} !=[n]_{q} \cdots[n]_{q}$ are the $q$-analogues of the integer and its factorial, respectively. The $q$-analogues of the gamma function are also given by [5]

$$
\Gamma_{q}(t)=\int_{0}^{\frac{1}{1-q}} x^{t-1} E_{q}(-q x) d_{q} x \text { and } \hat{\Gamma}_{q}(t)=\int_{0}^{\infty} x^{t-1} e_{q}(-x) d_{q} x
$$

The useful properties of the $q$-gamma functions $\Gamma_{q}$ and $\hat{\Gamma}_{q}$ are obtained in the literature as follows.

Theorem 1 Let $n \in \mathbb{N}$ and $t \in \mathbb{R}$. Then the following identities hold:
(i) $\Gamma_{q}(t+1)=[t]_{q} \Gamma_{q}(t)$ and $\Gamma_{q}(n+1)=[n]_{q}$ !,
(ii) $\Gamma_{q}(t+1)=\frac{1-q^{t}}{1-q} \Gamma_{q}(t)$
(iii) $\hat{\Gamma}_{q}(t+1)=q^{-t}[t]_{q} \hat{\Gamma}_{q}(t)$ and $\hat{\Gamma}_{q}(1)=1$,
(iv) $\hat{\Gamma}_{q}(n)=q^{\frac{-n(n-1)}{2}} \Gamma_{q}(n)$.

The $q$-analogues of the trigonometric functions $\sin x$ and $\cos x$ are given by (see, e.g., [4])

$$
\begin{array}{ll}
\sin _{q}(a t)=\sum_{n=0}^{\infty}(-1)^{n} \frac{q^{\frac{n(n+1)}{2}}}{[2 n+1]_{q} !} a^{2 n+1} t^{2 n+1}, & \cos _{q}(a t)=\sum_{n=0}^{\infty}(-1)^{n} \frac{q^{\frac{n(n-1)}{2}}}{[2 n]_{q} !} a^{2 n} t^{2 n} \\
\operatorname{Sin}_{q}(a t)=\sum_{n=0}^{\infty} \frac{(-1)^{n}}{[2 n+1]_{q} !} a^{2 n+1} t^{2 n+1} \text { and } \quad \operatorname{Cos}_{q}(a t)=\sum_{n=0}^{\infty} \frac{(-1)^{n}}{[2 n]_{q} !} a^{2 n} t^{2 n} .
\end{array}
$$

This paper is organized as follows. In Sect. 1, we present some preliminaries and notations that are very useful in the sequel. In Sect. 2, we apply the $q$-analogues of the Laplacetype integral operator to certain polynomials and functions of special-types. In Sect. 3, 
we apply the $q$-analogue $F_{2, q}$ to a certain class of differential operators of arbitrary order. In Sect. 4, we discuss the convolution product and establish a convolution theorem of the $F_{2, q}$ integral operator.

\section{$2 F_{2, q}$ and $S_{2, q}$ analogues of some $q$-special functions}

The Laplace integral operator, among various integral operators, is the most popular and widely used in several branches of engineering sciences and applied mathematics. The Laplace-type integral has been firstly defined by Yürekli and Sadek [19] and extended to a space of generalized functions by Al-Omari [20]. In [4], Ucar et al. have given the qanalogues of the Laplace-type integral operator of some elementary functions by making a free use of the identities of the $q$-Laplace integral operator. One of the goals of this paper is to provide the $q$-analogues of the Laplace-type integral operator, with a different approach, and derive results involving elementary functions and some other difference operators. The Laplace-type integral operator [19, (1.4)]

$$
L_{2}(\vartheta(x) ; y)=\int_{0}^{\infty} x \exp \left(-x^{2} y^{2}\right) \vartheta(x) d x
$$

has a close relation with the familiar Laplace integral operator given as

$$
L_{2}(\vartheta(x) ; y)=\frac{1}{2} L\left(\vartheta(\sqrt{x}) ; y^{2}\right) .
$$

The $q$-analogues of the Laplace-type integral operator were introduced by [4]

$$
{ }_{q} L_{2}(\vartheta(x) ; y)=\frac{1}{1-q^{2}} \int_{0}^{y^{-1}} x E_{q^{2}}\left(q^{2} y^{2} x^{2}\right) \vartheta(x) d_{q} x
$$

and

$$
{ }_{q} l_{2}(\vartheta(x) ; y)=\frac{1}{1-q^{2}} \int_{0}^{\infty} x e_{q^{2}}\left(-y^{2} x^{2}\right) \vartheta(x) d_{q} x
$$

provided $\operatorname{Re}(y)>0$. Here, we introduce two $q$-analogues of the Laplace-type integral operator in the standard way as

$$
F_{2, q}(\vartheta(x) ; y)=\int_{0}^{\infty} x \vartheta(x) E_{q}\left(-q x^{2} y^{2}\right) d_{q} x
$$

and

$$
S_{2, q}(\vartheta(x) ; y)=\int_{0}^{\infty} x \vartheta(x) e_{q}\left(-x^{2} y^{2}\right) d_{q} x,
$$

provided $\operatorname{Re}(y)>0$. In what follows, we make a use of Eq. (9) and Eq. (10) and provide a summary of some results related to the $F_{2, q}$ and $S_{2, q}$ analogues in the course of the following theorems.

Theorem 2 Let $\delta$ be a real number. Then the following hold:
(i) $\quad F_{2, q}\left(x^{2 \delta} ; y\right)=\frac{\Gamma_{q}(\delta+1)}{[2]_{q} y^{2 \delta+2}}$,
(ii) $S_{2, q}\left(x^{2 \delta} ; y\right)=\frac{\hat{\Gamma}_{q}(\delta+1)}{[2]_{q} y^{2 \delta+2}}$. 
Proof By aid of Eq. (9), we proceed by

$$
F_{2, q}\left(x^{2 \delta} ; y\right)=\int_{0}^{\infty} x^{2 \delta} E_{q}\left(-q x^{2} y^{2}\right) d_{q} x .
$$

Therefore, by the change of variables $x^{2} y^{2}=z$ we obtain

$$
F_{2, q}\left(x^{2 \delta} ; y\right)=\frac{1}{[2]_{q} y^{2 \delta+2}} \int_{0}^{\infty} z^{\delta} E_{q}(-q z) d_{q} z .
$$

Hence, the proof of the first part (i) of the theorem follows from Eq. (6). The proof of the second part (ii) follows by similar techniques. The proof of the theorem is completed.

Consequently, we state without proof the following straightforward corollary.

Corollary 3 The following identities hold:
(i) $\quad F_{2, q}\left(x^{2 n} ; y\right)=\frac{[n] !}{[2]_{q} y^{2 n+2}}$,
(ii) $S_{2, q}\left(x^{2 n} ; y\right)=q^{-\frac{(n-1)}{2}} \frac{[n]_{q} !}{[2]_{q} y^{2 n+2}}$.

Theorem 4 Let $\delta$ be a positive real number. Then the following identities hold:

(i) $\quad F_{2, q}\left(e_{q}\left(\delta x^{2}\right) ; y\right)=\frac{1}{[2]_{q}\left(y^{2}-\delta\right)} \quad\left(|\delta|<y^{2}\right)$,

(ii) $S_{2, q}\left(e_{q}\left(\delta x^{2}\right) ; y\right)=\sum_{n=0}^{\infty} \frac{\delta^{n}}{[2]_{q} y^{2 n+2}-q^{-n(n+1)}}$,

(iii) $\quad F_{2, q}\left(E_{q}\left(\delta x^{2}\right) ; y\right)=\sum_{n=0}^{\infty} \frac{q^{\frac{n(n-1)}{2}} \delta^{n}}{[2]_{q} y^{2 n+2}}$,

(iv) $S_{2, q}\left(E_{q}\left(\delta x^{2}\right) ; y\right)=\sum_{n=0}^{\infty} \frac{-q^{n}}{[2]_{q}[n]_{q} y^{2 n+2}} \delta^{n}$.

Proof Let $\delta$ be a positive real number. Then, by employing Eq. (9) and Eq. (5) we write

$$
F_{2, q}\left(e_{q}\left(\delta x^{2}\right) ; y\right)=\sum_{n=0}^{\infty} \frac{\delta^{n}}{[n]_{q} !} \int_{0}^{\infty} x^{2 n+1} E_{q}\left(-q x^{2} y^{2}\right) d_{q} x .
$$

By using the change of variables $z=x^{2} y^{2}$, the above equation yields

$$
F_{2, q}\left(e_{q}\left(\delta x^{2}\right) ; y\right)=\sum_{n=0}^{\infty} \frac{\delta^{n}}{[n]_{q} !} \int_{0}^{\infty} \frac{z^{n}}{[2]_{q} y^{2 n+2}} E_{q}(-q z) d_{q} z
$$

Hence, by invoking the definition of the gamma function given by Eq. (6), we obtain

$$
F_{2, q}\left(e_{q}\left(\delta x^{2}\right) ; y\right)=\sum_{n=0}^{\infty} \frac{\delta^{n}}{[n]_{q} !} \frac{1}{[2]_{q} y^{2 n+2}} \Gamma_{q}(n+1) .
$$


Therefore, by applying Theorem 1, Eq. (11) leads to the geometric series

$$
F_{2, q}\left(e_{q}\left(\delta x^{2}\right) ; y\right)=\sum_{n=0}^{\infty} \frac{\delta^{n}}{[2]_{q} y^{2 n+2}}=\frac{1}{[2]_{q}\left(y^{2}-\delta\right)} \quad\left(|\delta|<y^{2}\right)
$$

This proves the first part of the theorem. By following a like approach, we get

$$
S_{2, q}\left(e_{q}\left(\delta x^{2}\right) ; y\right)=\sum_{n=0}^{\infty} \frac{\delta^{n}}{[2]_{q} y^{2 n+2}[n]_{q} !} \hat{\Gamma}_{q}(n+1) .
$$

This, indeed, proves the second part of the theorem. The proof of the third part (iii) follows from a similar technique. To establish the fourth part (iv) of the theorem, we make use of the definition of the $q$-analogue $E_{q}$ to write

$$
F_{2, q}\left(E_{q}\left(\delta x^{2}\right) ; y\right)=\sum_{n=0}^{\infty} \frac{q^{\frac{n(n-1)}{2}}}{[n]_{q} !} \delta^{n} \int_{0}^{\infty} x^{2 x+1} e_{q}\left(-x^{2} y^{2}\right) d_{q} x
$$

By applying the change of variables $z=x^{2} y^{2}$, our previous equation becomes

$$
F_{2, q}\left(E_{q}\left(\delta x^{2}\right) ; y\right)=\sum_{n=0}^{\infty} \frac{q^{\frac{n(n-1)}{2}}}{[n]_{q} !} \frac{\delta^{n}}{[2]_{q} y^{2 n+2}} \hat{\Gamma}_{q}(n+1) .
$$

Hence, by using Part (iv) of Theorem 1, the equation can be put into the form

$$
F_{2, q}\left(E_{q}\left(\delta x^{2}\right) ; y\right)=\sum_{n=0}^{\infty} \frac{q^{\frac{n(n-1)}{2}} \delta^{n}}{[n]_{q} ![2]_{q} y^{2 n+2}} q^{\frac{-n(n+1)}{2}} \Gamma_{q}(n) .
$$

Therefore, simple computations yield

$$
F_{2, q}\left(E_{q}\left(\delta x^{2}\right) ; y\right)=\sum_{n=0}^{\infty} \frac{-q^{n}}{[2]_{q}[n]_{q} y^{2 n+2}} \delta^{n} .
$$

Hence, the proof of this theorem is therefore completed.

Theorem 5 Let $\delta$ be a positive real number. Then the following identities hold:

(i) $F_{2, q}\left(\operatorname{Cos}_{q}\left(\delta x^{2}\right) ; y\right)=\frac{y^{2}}{[2]_{q}\left(y^{4}+\delta^{2}\right)} \quad\left(\delta^{2}<y^{4}\right)$,

(ii) $S_{2, q}\left(\operatorname{Cos}_{q}\left(\delta x^{2}\right) ; y\right)=\frac{1}{[2]_{q} y^{2}} \sum_{n=0}^{\infty}(-1)^{n} q^{\frac{n(n-1)}{2}} \frac{\delta^{2 n}}{y^{4 n}}$,

(iii) $F_{2, q}\left(\cos _{q}\left(\delta x^{2}\right) ; y\right)=\frac{1}{[2]_{q} y^{2}} \sum_{n=0}^{\infty} q^{\frac{n(n-1)}{2}}\left(\frac{-\delta^{2}}{y^{4}}\right)^{n}$,

(iv) $S_{2, q}\left(\cos _{q}\left(\delta x^{2}\right) ; y\right)=\sum_{n=0}^{\infty}(-1)^{n} \frac{q^{n\left(\frac{n-1}{2}\right)}}{[2]_{q} y^{4 n+2}} \quad(2 n>-1)$. 
Proof Let $\delta$ be a positive real number. Then, by the definition of $F_{2, q}$ and the definition of $\mathrm{Cos}_{q}$, we write

$$
F_{2, q}\left(\operatorname{Cos}_{q}\left(\delta x^{2}\right) ; y\right)=\sum_{n=0}^{\infty} \frac{(-1)^{n}}{[2 n]_{q} !} \delta^{2 n} \int_{0}^{\infty} x^{4 n+1} E_{q}\left(-q x^{2} y^{2}\right) d_{q} x
$$

By making the change of variables $z=x^{2} y^{2}$ we derive

$$
\begin{aligned}
F_{2, q}\left(\operatorname{Cos}_{q}\left(\delta x^{2}\right) ; y\right) & =\sum_{n=0}^{\infty} \frac{(-1)^{n}}{[2 n]_{q} !} \frac{\delta^{2 n}}{[2]_{q} y^{4 n+2}} \int_{0}^{\infty} z^{2 n} E_{q}(-q z) d_{q} z \\
& =\sum_{n=0}^{\infty} \frac{(-1)^{n}}{[2 n]_{q} !} \frac{\delta \Gamma_{q}(2 n+1)}{[2]_{q} y^{4 n+2}}
\end{aligned}
$$

Consequently, by employing Theorem 1, Eq. (12) turns out to be in the form of the geometric series

$$
F_{2, q}\left(\operatorname{Cos}_{q}\left(\delta x^{2}\right) ; y\right)=\frac{1}{[2]_{q} y^{2}} \sum_{n=0}^{\infty}\left(\frac{-\delta^{2}}{y^{4}}\right)^{n}
$$

Therefore, the convergence condition of the geometric series shows that

$$
F_{2, q}\left(\operatorname{Cos}_{q}\left(\delta x^{2}\right) ; y\right)=\frac{y^{2}}{[2]_{q}\left(y^{2}+\delta^{2}\right)} \quad\left(\delta^{2}<y^{4}\right) .
$$

The proof of Part (i) is therefore finished. The proof of the Part (ii) follows from Theorem 1, Part (v) and a similar argument to that employed in the first part. Once again, an argument similar to the argument we have employed for Part (i) establishes Part (iii). Finally, Part (iv) is a straightforward result of Theorem 2. This finishes the proof of the theorem.

A similar statement to the statement of Theorem 5 can be read as follows.

Theorem 6 Let $\delta$ be a positive real number. Then the following identities hold true:

(i) $\quad F_{2, q}\left(\operatorname{Sin}_{q}\left(\delta x^{2}\right) ; y\right)=\frac{\delta}{[2]_{q}\left(y^{4}+\delta^{2}\right)} \quad\left(\delta^{2}<y^{4}\right)$,

(ii) $S_{2, q}\left(\operatorname{Sin}_{q}\left(\delta x^{2}\right) ; y\right)=\frac{\delta}{[2]_{q} y^{4}} \sum_{n=0}^{\infty}(-1)^{n} q^{\frac{-n(n-1)}{2}} \frac{\delta^{2 n}}{y^{4 n}}$,

(iii) $\quad F_{2, q}\left(\sin _{q}\left(\delta x^{2}\right) ; y\right)=\sum_{n=0}^{\infty}(-1)^{n} q^{\frac{-n(n-1)}{2}} \frac{\delta^{2 n+1}}{[2]_{q}} y^{-4 n+4}$,

(iv) $S_{2, q}\left(\sin _{q}\left(\delta x^{2}\right) ; y\right)=\sum_{n=0}^{\infty}(-1)^{n} \frac{q^{n}}{[2 n+1]_{q}} \frac{y^{-4 n-4}}{[2]_{q}} \quad(2 n>-1)$.

The proof of this theorem follows from definitions and a similar argument to that we already checked for Theorem 5. Details are therefore omitted. 
Now, by following the usual notations of [16] and the facts that

$$
\cosh _{q} x=\frac{e_{q}(x)+e_{q}(-x)}{2} \text { and } \sinh _{q} x=\frac{e_{q}(x)-e_{q}(-x)}{2}
$$

we state the following straightforward corollary.

Corollary 7 Let $\delta$ be positive real number. Then we have
(i) $\quad F_{2, q}\left(\cosh _{q}\left(\delta x^{2}\right) ; y\right)=\frac{y^{2}}{[2]_{q}\left(y^{2}-\delta^{2}\right)}$,
(ii) $\quad F_{2, q}\left(\sinh _{q}\left(\delta x^{2}\right) ; y\right)=\frac{\delta}{[2]_{q}\left(y^{4}-\delta^{2}\right)}$.

The proof of this corollary directly follows from Eq. (13), Theorems 5 and 6. Details are omitted.

\section{$3 F_{2, q}$ of $q$-differential operators}

We devote this section to computations related to the $F_{2, q}$ integral and some differential operators. First of all, we derive the following theorem.

Theorem 8 Let $y>0$. Then we have

$$
D_{x, q} E_{q}\left(-q x^{2} y^{2}\right)=-x y^{2} \sum_{n=0}^{\infty}(-1)^{n} q^{\frac{(n+1) n}{2}}\left(1+q^{n+1}\right) y^{2 n} x^{2 n}
$$

Proof By Eq. (4), we write

$$
D_{x, q} E_{q}\left(-q x^{2} y^{2}\right)=\sum_{n=1}^{\infty}(-1)^{n} \frac{q^{\frac{(n-1) n}{2}}}{[n]_{q} !}[2 n]_{q} q^{n} y^{2 n} x^{2 n-1} .
$$

But simple computations then yield

$$
[2 n]_{q}=\frac{1-q^{2 n}}{1-q}=\frac{1-\left(q^{n}\right)^{2}}{1-q}=[n]_{q}\left(1+q^{n}\right) .
$$

Hence, shifting the lower bound of the above summation implies

$$
\begin{aligned}
D_{x, q} E_{q}\left(-q x^{2} y^{2}\right) & =\sum_{n=0}^{\infty}(-1)^{n+1} \frac{q^{\frac{(n-1) n}{2}}}{[n]_{q} !}\left(1+q^{n+1}\right) y^{2 n+2} x^{2 n+1} \\
& =-x y^{2} \sum_{n=0}^{\infty}(-1)^{n} q^{\frac{(n+1) n}{2}}\left(1+q^{n+1}\right) y^{2 n} x^{2 n}
\end{aligned}
$$

The proof of the theorem is therefore finished.

Theorem 9 Let $\Delta_{q}(x)=\frac{1}{x} D_{x, q}$. Then we have

$$
F_{2, q}\left(\Delta_{q} \vartheta ; y\right)=-\vartheta(0)+\frac{1+q}{q^{2}} y^{2} F_{2, q}\left(\vartheta ; \frac{y}{q}\right) .
$$


Proof By the $q$-integration by parts given by Eq. (3), we write

$$
\begin{aligned}
F_{2, q}\left(\Delta_{q} \vartheta ; y\right) & =\int_{0}^{\infty} D_{x, q} \vartheta(x) e_{q}\left(-q x^{2} y^{2}\right) d_{q} x \\
& =-f(0)-\int_{0}^{\infty} \vartheta(q x) D_{q} E_{q}\left(-q x^{2} y^{2}\right) d_{q} x .
\end{aligned}
$$

Hence, by applying Theorem 8, Eq. (15) can be expressed as

$$
F_{2, q}\left(\Delta_{q} \vartheta ; y\right)=-\vartheta(0)+y^{2} \int_{0}^{\infty} x \vartheta(q x) \sum_{n=0}^{\infty}(-1)^{n} \frac{q^{\frac{(n+1) n}{2}}}{[n]_{q} !}\left(1+q^{n+1}\right) y^{2 x} x^{2 x} d_{q} x .
$$

Therefore, by changing the variables as $q x=z\left(d_{q} x=\frac{1}{q} d_{q} z\right)$, we transfer Eq. (16) into the form

$$
\begin{aligned}
F_{2, q}\left(\Delta_{q} \vartheta ; y\right)= & -\vartheta(0)+y^{2} \int_{0}^{\infty} q^{-1} z \vartheta(z) \sum_{n=0}^{\infty}(-1)^{n} \frac{q^{\frac{(n+1) n}{2}}}{[n]_{q} !}\left(1+q^{n+1}\right) y^{2 n} z^{2 n} q^{-1} d_{q} z \\
= & -\vartheta(0)+y^{2}+q^{-2} y^{2} \int_{0}^{\infty} z \vartheta(z) \sum_{n=0}^{\infty}(-1)^{n} \frac{q^{\frac{(n+1) n}{2}}}{[n]_{q} !} y^{2 n} q^{-2 n} d_{q} z \\
& +q^{-2} y^{2} \int_{0}^{\infty} z \vartheta(z) \sum_{n=0}^{\infty}(-1)^{n} \frac{q^{\frac{(n+1) n}{2}}}{[n]_{q} !} q^{n+1} y^{2 n} q^{-2 n} z^{2 n} d_{q} z .
\end{aligned}
$$

Thus, by multiplying the previous equation by $q^{-n} q^{n}$, we obtain

$$
\begin{aligned}
F_{2, q}\left(\Delta_{q} \vartheta ; y\right)= & -\vartheta(0)+q^{-2} y^{2} \int_{0}^{\infty} z \vartheta(z) \sum_{n=0}^{\infty}(-1)^{n} \frac{q^{\frac{(n+1) n}{2}}}{[n]_{q} !} q^{-3 n} y^{2 n} q^{n} z^{2 n} d_{q} z \\
& +q^{-2} y^{2} \int_{0}^{\infty} z \vartheta(z) \sum_{n=0}^{\infty}(-1)^{n} \frac{q^{\frac{(n+1) n}{2}}}{[n]_{q} !} q^{n+1} q^{-3 n} y^{2 n} q^{n} z^{2 n} d_{q} z .
\end{aligned}
$$

Thus, this equation can be nicely expressed as

$$
\begin{aligned}
F_{2, q}\left(\Delta_{q} \vartheta ; y\right)= & -\vartheta(0)+q^{-2} y^{2} \int_{0}^{\infty} z \vartheta(z) \sum_{n=0}^{\infty} \frac{(-1)^{n}}{[n]_{q} !} q^{\frac{(n-1) n}{2}}\left(\frac{y^{2}}{q^{2}}\right)^{n} q^{n} z^{2 n} d_{q} z \\
& +q^{-2} y^{2} \int_{0}^{\infty} z \vartheta(z) \sum_{n=0}^{\infty} \frac{(-1)^{n}}{[n]_{q} !} q^{\frac{(n-1) n}{2}} \cdot\left(\frac{y^{2}}{q^{2}}\right)^{n} q^{n} z^{2 n} q d_{q} z .
\end{aligned}
$$

Finally, the preceding equation can be written as

$$
\begin{aligned}
F_{2, q}\left(\Delta_{q} \vartheta ; y\right)= & -\vartheta(0)+q^{-2} y^{2} \int_{0}^{\infty} z \vartheta(z) E_{q}\left(-q\left(\frac{y}{q}\right)^{2 n} z^{2 n}\right) d_{q} z \\
& +q^{-1} y^{2} \int_{0}^{\infty} z \vartheta(z) E_{q}\left(-q\left(\frac{y}{q}\right)^{2 n} z^{2 n}\right) d_{q} z \\
= & -\vartheta(0)+q^{-2} y^{2} L_{2, q}\left(\vartheta ; \frac{y}{q}\right)+q^{-1} y^{2} F_{2, q}\left(\vartheta ; \frac{y}{q}\right) .
\end{aligned}
$$

This finishes the proof of the theorem. 
Theorem 10 Let y be a positive real number. Then we have

$$
F_{2, q}\left(\Delta_{q}^{2} ; y\right)=-\left(\Delta_{q} \vartheta\right)(0)-\frac{1+q}{q^{2}} y^{2} \vartheta(0)+\frac{(1+q)^{2}}{\left(q^{2}\right)^{2}} \frac{y^{2}}{q^{2}} y^{2} F_{2, q}\left(\vartheta, \frac{y}{q^{2}}\right)
$$

Proof Let $y>0$. Then, by using Theorem 9, we obtain

$$
\begin{aligned}
F_{2, q}\left(\Delta_{q}^{2} ; y\right) & =-\left(\Delta_{q} \vartheta\right)(0)+\frac{1+q}{q^{2}} y^{2}\left(-\vartheta(0)+\frac{1+q}{q^{2}}\left(\frac{y}{q}\right)^{2} F_{2, q}\left(\vartheta, \frac{y}{q^{2}}\right)\right) \\
& =-\left(\Delta_{q} \vartheta\right)(0)-\frac{1+q}{q^{2}} y^{2} \vartheta(0)+\left(\frac{1+q}{q^{2}}\right)^{2} \frac{y^{2}}{q^{2}} y^{2} F_{2, q}\left(\vartheta ; \frac{y}{q^{2}}\right) .
\end{aligned}
$$

Hence, the proof of the theorem is finished.

Theorem 11 Let y be a positive real number. Then we have

$$
\begin{aligned}
F_{2, q}\left(\Delta_{q}^{3} \vartheta ; y\right)= & -\left(\Delta_{q}^{2} \vartheta\right)(0)-\frac{1+q}{q^{2}} y^{2} \Delta_{q} \vartheta(0)-\frac{(1+q)^{2}}{\left(q^{2}\right)^{2}} \frac{y^{2}}{q^{2}} y^{2} \vartheta(0) \\
& +\frac{(1+q)^{3}}{\left(q^{2}\right)^{3}} \frac{y^{2}}{q^{4}} \frac{y^{2}}{q^{2}} y^{2} F_{2, q}\left(\vartheta, \frac{y}{q^{3}}\right) .
\end{aligned}
$$

Proof By taking into account Theorem 9 and using simple computations, we have

$$
F_{2, q}\left(\Delta_{q}^{3} \vartheta ; y\right)=F_{2, q}\left(\Delta_{q} \Delta_{q}^{2} \vartheta ; y\right)=-\Delta_{q}^{2} \vartheta(0)+\frac{1+q}{q^{2}} y^{2} F_{2, q}\left(\Delta^{2} \vartheta ; \frac{y}{q}\right)
$$

By utilizing Theorem 10, Eq. (18) together with computations reveals

$$
\begin{aligned}
F_{2, q}\left(\Delta_{q}^{3} \vartheta ; y\right)= & -\Delta_{q}^{2} \vartheta(0)+\frac{1+q}{q^{2}} y\left(-\frac{1+q}{q^{2}}\left(\frac{y}{q}\right)^{2} \vartheta(0)-\Delta_{q} \vartheta(0)\right) \\
& +\frac{1+q}{q^{2}} y\left(\frac{(1+q)^{2}}{\left(q^{2}\right)^{2}} \frac{y^{2}}{q^{2}-q^{2}} \frac{y^{2}}{q^{2}} F_{2, q}\left(\vartheta, \frac{y}{q^{3}}\right)\right) \\
= & -\left(\Delta_{q}^{2} \vartheta\right)(0)-\frac{1+q}{q^{2}} y^{2} \Delta_{q} \vartheta(0)-\frac{(1+q)^{2}}{\left(q^{2}\right)^{2}} \frac{y^{2}}{q^{2}} y^{2} \vartheta(0) \\
& +\frac{(1+q)^{3}}{\left(q^{2}\right)^{3}} \frac{y^{2}}{q^{4}} \frac{y^{2}}{q^{2}} y^{2} F_{2, q}\left(\vartheta, \frac{y}{q^{3}}\right) .
\end{aligned}
$$

This finishes the proof of the theorem.

Theorem 12 Let $y$ be a positive real number. Then we have

$$
\begin{aligned}
F_{2, q}\left(\Delta_{q}^{4} \vartheta ; y\right)= & -\Delta_{q}^{3} \vartheta(0)-\frac{1+q}{q^{2}} y^{2} \Delta_{q}^{2} \vartheta(0)-\left(\frac{1+q}{q^{2}}\right)^{2}\left(\frac{y}{q}\right)^{2} y^{2} \Delta_{q} \vartheta(0) \\
& -\left(\frac{1+q}{q^{2}}\right)^{3} \frac{y^{2}}{q^{4}} \frac{y^{2}}{q^{2}} \cdot y^{2} \vartheta(0) \\
& +\left(\frac{1+q}{q^{2}}\right)^{4} \frac{y^{2}}{q^{6}} \frac{y^{2}}{q^{4}} \frac{y^{2}}{q^{2}} y^{2} F_{2, q}\left(\vartheta ; \frac{y}{q^{4}}\right)
\end{aligned}
$$


Proof Let $y>0$ be given arbitrary. Then, by employing Theorem 10, we derive that

$$
\begin{aligned}
F_{2, q}\left(\Delta_{q}^{4} \vartheta ; y\right)= & -\Delta_{q}^{3} \vartheta(0)+\frac{1+q}{q^{2}} y^{2} L_{2, q}\left(\Delta_{q}^{3} \vartheta ; \frac{y}{q}\right) \\
= & -\Delta_{q}^{3} \vartheta(0)+\frac{1+q}{q^{2}} y^{2}\left(-\Delta_{q}^{2} \vartheta(0)-\frac{1+q}{q^{2}}\left(\frac{y}{q}\right)^{2} \Delta_{q} \vartheta(0)\right) \\
& -\frac{1+q}{q^{2}} y^{2}\left(\left(\frac{1+q}{q^{2}}\right)^{2} \frac{y^{2}}{q^{4}} \frac{y^{2}}{q^{2}} \vartheta(0)+\left(\frac{1+q}{q^{2}}\right)^{3} \frac{y^{2}}{q^{6}} \frac{y^{2}}{q^{4}} \frac{y^{2}}{q^{2}} F_{2, q}\left(\vartheta, \frac{y}{q^{4}}\right)\right) .
\end{aligned}
$$

Therefore, further simplifications on the above equation yield

$$
\begin{aligned}
F_{2, q}\left(\Delta_{q}^{4} \vartheta ; y\right)= & -\Delta_{q}^{3} \vartheta(0)-\frac{1+q}{q^{2}} y^{2} \Delta_{q}^{2} \vartheta(0)-\left(\frac{1+q}{q^{2}}\right)^{2}\left(\frac{y}{q}\right)^{2} y^{2} \Delta_{q} \vartheta(0) \\
& \times\left(\frac{1+q}{q^{2}}\right)^{3} \frac{y^{2}}{q^{4}} \frac{y^{2}}{q^{2}} y^{2} \vartheta(0)+\left(\frac{1+q}{q^{2}}\right)^{4} y^{2} \frac{y^{2}}{q^{6}} \frac{y^{2}}{q^{4}} \frac{y^{2}}{q^{2}} F_{2, q}\left(\vartheta, \frac{y}{q^{4}}\right) .
\end{aligned}
$$

Hence, the proof of the theorem is finished.

By following techniques similar to the techniques already used for Theorems 10-12, we reach the following result.

Corollary 13 Let $y>0$. Then we have

$$
\begin{aligned}
F_{2, q}\left(\Delta_{q}^{n} \vartheta ; y\right)= & -\sum_{j=1}^{n-1}\left(\frac{1+q}{q^{2}}\right)^{n-j-1} \prod_{k=1}^{n-j-1} \frac{y^{2}}{q^{2 k-2}} \Delta_{q}^{j} \vartheta(0)-\left(\frac{1+q}{q^{2}}\right)^{n-1} \\
& \times \prod_{k=1}^{n-1} \frac{y^{2}}{q^{2 k-2}} \vartheta(0)+\left(\frac{1+q}{q^{2}}\right)^{n} \prod_{k=1}^{n} \frac{y^{2}}{q^{2 k-2}} F_{2, q}\left(\vartheta, \frac{y}{q^{n}}\right) .
\end{aligned}
$$

Let us now check the following differentiation formula.

Theorem 14 Let y be a positive real number. Then we have

$$
D_{x, q} e_{q}\left(-x^{2} y^{2}\right)=-y^{2} x \sum_{n=0}^{\infty} \frac{(-1)^{n}}{[n]_{q} !}\left(1+q^{n+1}\right) y^{2 n} x^{2 n} .
$$

Proof Let $y$ be a positive real number. Then, from the definitions, we have

$$
D_{x, q} e_{q}\left(-x^{2} y^{2}\right)=\sum_{n=1}^{\infty}(-1)^{n} \frac{[2 n]_{q}}{[n]_{q} !} y^{2 n} x^{2 n-1}
$$

But, as earlier, we know that

$$
[2 n]_{q}=\frac{1-q^{2 n}}{1-q}=\frac{1-\left(q^{n}\right)^{2}}{1-q}=[n]_{q}\left(1+q^{n}\right) .
$$


Hence, invoking these results in the previous summation gives

$$
\begin{aligned}
D_{x, q} e_{q}\left(-x^{2} y^{2}\right) & =\sum_{n=1}^{\infty}(-1)^{n} \frac{[n]_{q}}{[n]_{q} !}\left(1+q^{n}\right) y^{2 n} x^{2 n-1} \\
& =\sum_{n=1}^{\infty} \frac{(-1)^{n}}{[n-1]_{q} !}\left(1+q^{n}\right) y^{2 n} x^{2 n-1} \\
& =\sum_{n=0}^{\infty} \frac{(-1)^{n+1}}{[n]_{q} !}\left(1+q^{n+1}\right) y^{2 n+2} x^{2 n+1} \\
& =-y^{2} x \sum_{n=0}^{\infty} \frac{(-1)^{n}}{[n]_{q} !}\left(1+q^{n+1}\right) y^{2 n} x^{2 n} .
\end{aligned}
$$

This finishes the proof of the theorem.

Theorem 15 Let $\Delta_{q}(x)=\frac{1}{x} D_{x, q}$ be given. Then we have

$$
S_{2, q}\left(\Delta_{q} \vartheta ; y\right)=-\vartheta(0)+q^{-1} y^{2} S_{2, q}\left(\vartheta ; \frac{y}{q}\right)+y^{2} S_{2, q}\left(\vartheta ; \frac{y}{\sqrt{q}}\right) .
$$

Proof By making use of Eq. (9) and employing Eq. (3), we establish that

$$
\begin{aligned}
S_{2, q}\left(\Delta_{q} \vartheta ; y\right) & =\int_{0}^{\infty} D_{x, q} \vartheta(x) e_{q}\left(-x^{2} y^{2}\right) d_{q} x \\
& =\left.\vartheta(x) e_{q}\left(-x^{2} y^{2}\right)\right|_{0} ^{\infty}-\int_{0}^{\infty} \vartheta(q x) D_{x, q} e_{q}\left(-x^{2} y^{2}\right) d_{q} x
\end{aligned}
$$

Therefore, substituting the integral bounds in the first part suggests we have

$$
S_{2, q}\left(\Delta_{q} \vartheta ; y\right)=-\vartheta(0)-\int_{0}^{\infty} \vartheta(q x) D_{x, q} e_{q}\left(-x^{2} y^{2}\right) d_{q} x
$$

Hence, by virtue of Theorem 10, Eq. (19) reveals

$$
S_{2, q}\left(\Delta_{q} \vartheta ; y\right)=-\vartheta(0)+y^{2} \int_{0}^{\infty} \vartheta(q x) x \sum_{n=0}^{\infty} \frac{(-1)^{n}}{[n]_{q} !}\left(1+q^{n+1}\right) y^{2 n} x^{2 n} d_{q} x .
$$

The change of variables $q x=z$ yields $d_{q} x=\frac{1}{q} d_{q} z$. Therefore, the above equation together with certain technical computations implies

$$
\begin{aligned}
S_{2, q}\left(\Delta_{q} \vartheta ; y\right) & =-\vartheta(0)+y^{2} \int_{0}^{\infty} \vartheta(z) \frac{z}{q} \sum_{n=0}^{\infty} \frac{(-1)^{n}}{[n]_{q} !}\left(1+q^{n+1}\right) y^{2 n} \frac{z^{2 n}}{q^{2 n}} \frac{1}{q} d_{q} z \\
& =-\vartheta(0)+y^{2} \int_{0}^{\infty} \vartheta(z) \sum_{n=0}^{\infty} \frac{(-1)^{n}}{[n]_{q} !} \frac{\left(1+q^{n+1}\right)}{q^{2(n+1)}} y^{2 n} z^{2 n} d_{q} z \\
& =-\vartheta(0)+y^{2} \int_{0}^{\infty} z \vartheta(z) \sum_{n=0}^{\infty} \frac{(-1)^{n}}{[n]_{q} !}\left(q^{-2 n-1-n}+q\right) y^{2 n} z^{2 n} d_{q} z
\end{aligned}
$$




$$
\begin{aligned}
= & -\vartheta(0)+q^{-1} y^{2} \int_{0}^{\infty} z \vartheta(z) \sum_{n=0}^{\infty} \frac{(-1)^{n}}{[n]_{q} !}\left(\frac{y^{2}}{q^{2}}\right)^{n} z^{2 n} d_{q} z \\
& +y^{2} \int_{0}^{\infty} z \vartheta(z) \sum_{n=0}^{\infty} \frac{(-1)^{n}}{[n]_{q} !}\left(\frac{y^{2}}{\sqrt{q}}\right)^{n} z^{2 n} d_{q} z \\
= & -\vartheta(0)+q^{-1} y^{2} \int_{0}^{\infty} z \vartheta(z) e_{q}\left(\frac{y^{2}}{q^{2}} z^{2}\right) d_{q} z \\
& +y^{2} \int_{0}^{\infty} z \vartheta(z) e_{q}\left(\frac{y^{2}}{q^{2}} z^{2}\right) z^{2 n} d_{q} z \\
= & -\vartheta(0)+q^{-1} y^{2} S_{2, q}\left(\vartheta ; \frac{y}{q}\right)+y^{2} S_{2, q}\left(\vartheta ; \frac{y}{\sqrt{q}}\right) .
\end{aligned}
$$

Hence, the proof of this theorem is completed.

\section{$4 F_{2, q}$ of $q$-convolution products}

In this section we focus on giving a convolution theorem for the $F_{2, q}$ integral operator. For, let us assume $\vartheta(t)=t^{2 \delta}$ and $\theta(t)=t^{2 \gamma-1}, \delta, \gamma>0$. Then the $q$-convolution product is defined for $\vartheta$ and $\theta$ as (see [15, Eq. (44)])

$$
(\vartheta * \theta)_{q}(t)=\int_{0}^{t} \vartheta(x) \theta(t-q x) d_{q} x
$$

provided the integral exists. The following is a very useful property.

Theorem 16 Let $\vartheta(t)=t^{2 \delta}$ and $\theta(t)=t^{2 \gamma-1}, \delta, \gamma>0$. Then we have

$$
(\vartheta * \theta)_{q}(t)=t^{2 \delta+2 \gamma} \frac{\Gamma_{q}(2 \delta+1) \Gamma_{q}(2 \gamma)}{\Gamma_{q}(2 \delta+2 \gamma+1)} .
$$

Proof By using the integral equation given by Eq. (20), we get

$$
(\vartheta * \theta)_{q}(t)=\int_{0}^{t} x^{2 \delta}(t-q x)^{2 \gamma-1} d_{q} x .
$$

The change of variables $x=t z$ transforms the equation into the form

$$
\begin{aligned}
(\vartheta * \theta)_{q}(t) & =\int_{0}^{1} t^{2+1} z^{2 \delta}(t-q t z)^{2 \gamma-1} d_{q} z \\
& =\int_{0}^{1} t^{2+1} z^{2 \delta} t^{2 \gamma-1}(1-q z)^{2 \gamma-1} d_{q} z \\
& =t^{2 \delta+2 \gamma} \int_{0}^{1} z^{2 \delta}(1-q z)^{2 \gamma-1} d_{q} z \\
& =t^{2 \delta+2 \gamma} B_{q}(2 \delta+1,2 \gamma),
\end{aligned}
$$

where $B_{q}$ is the $q$-analogue of the Beta function,

$$
B_{q}(\delta, \gamma)=\int_{0}^{1} t^{\delta}(1-q t)^{\gamma-1} d_{q} t
$$


Hence, by the well-known formula $B_{q}(\delta, \gamma)=\frac{\Gamma_{q}(\delta) \Gamma_{q}(\gamma)}{\Gamma_{q}(\delta+\gamma)}$, we get

$$
(\vartheta * \theta)_{q}(t)=t^{2 \delta+2 \gamma} \frac{\Gamma_{q}(2 \delta+1) \Gamma_{q}(2 \gamma)}{\Gamma_{q}(2 \delta+2 \gamma+1)} .
$$

This finishes the proof of the above result.

Now, by the aid of Theorem 16, we establish the following convolution theorem.

Theorem 17 Let $\delta, \gamma>0, \delta+\gamma>-\frac{1}{2}, \vartheta(x)=x^{2 \delta}$ and $\theta(x)=x^{2 \gamma-1}$. Then we have

$$
F_{2, q}\left((\vartheta * \theta)_{q}(x) ; y\right)=\frac{1}{[2]_{q}} \frac{\Gamma_{q}(2 \delta+1) \Gamma_{q}(2 \gamma) \Gamma_{q}(\delta+\gamma+1)}{\Gamma_{q}(2 \delta+2 \gamma+1)} y^{-2 \delta-2 \gamma-2}
$$

Proof Let $\delta, \gamma>0, \vartheta(x)=x^{2 \delta}$ and $\theta(x)=x^{2 \gamma-1}$ be given. Then we obtain

$$
\begin{aligned}
F_{2, q}\left((\vartheta * \theta)_{q} ; y\right) & =\int_{0}^{\infty}(\vartheta * \theta)_{q}(x) x E_{q}\left(-q x^{2} y^{2}\right) d_{q} x \\
& =\frac{\Gamma_{q}(2 \delta+1) \Gamma_{q}(2 \gamma)}{\Gamma_{q}(2 \delta+2 \gamma+1)} \int_{0}^{\infty} x^{2 \delta+2 \gamma} x E_{q}\left(-q x^{2} y^{2}\right) d_{q} x .
\end{aligned}
$$

Hence, from above, we obtain

$$
F_{2, q}\left((\vartheta * \theta)_{q} ; y\right)=\frac{\Gamma_{q}(2 \delta+1) \Gamma_{q}(2 \gamma)}{\Gamma_{q}(2 \delta+2 \gamma+1)} F_{2, q}\left(x^{2(\delta+\gamma)} ; y\right) .
$$

Therefore, by aid of Theorem 2, we put Eq. (22) into the form

$$
F_{2, q}\left((\vartheta * \theta)_{q} ; y\right)=\frac{\Gamma_{q}(2 \delta+1) \Gamma_{q}(2 \gamma)}{\Gamma_{q}(2 \delta+2 \gamma+1)} \frac{\Gamma_{q}(\delta+\gamma+1)}{[2]_{q} y^{2 \delta+2 \gamma+2}},
$$

$\delta, \gamma>0, \delta+\gamma>-\frac{1}{2}$.

This finishes the proof of the theorem.

\section{Conclusion}

The $q$-Laplace-type integral operator was applied to certain polynomials and various functions of $q$-trigonometric types. Such results were extended to $q$-hyperbolic functions and some other $q$-differential operators. By using $q$-differentiation formulas, several derivatives of the $q$-Laplace-type integral operator were obtained. On top of that, some related formulas as well as a convolution theorem were also discussed in detail.

Acknowledgements

The author would like to thank his parents, friends and Springer Nature for their support.

Funding

No funding sources to be declared.

Availability of data and materials

Please contact author for data requests. 
Authors' contributions

The author was the only one to write this paper. The author read and approved the final manuscript.

\section{Publisher's Note}

Springer Nature remains neutral with regard to jurisdictional claims in published maps and institutional affiliations.

Received: 14 May 2020 Accepted: 31 July 2020 Published online: 08 August 2020

\section{References}

1. Jackson, F.H.: On q-definite integrals. Q. J. Pure Appl. Math. 41, 193-203 (1910)

2. Abdi, W.H.: On q-Laplace transforms. Proc. Natl. Acad. Sci., India 29, 389-408 (1961)

3. Purohit, S.D., Kalla, S.L.: On q-Laplace transforms of the $q$-Bessel functions. Fract. Calc. Appl. Anal. 10(2), 189-196 (2007)

4. Ucar, F., Albayrak, D.: On q-Laplace type integral operators and their applications. J. Differ. Equ. Appl. 18, 1001-1014 (2012)

5. Albayrak, D., Purohit, S.D., Ucar, F:. On q-analogues of Sumudu transform. An. Ştiinţ. Univ. 'Ovidius' Constanţa 21(1), 239-260 (2013)

6. Yadav, R., Purohit, S.D.: On applications of Weyl fractional 9 -integral operator to generalized basic hypergeometric functions. Kyungpook Math. J. 46, 235-245 (2006)

7. Fitouhi, A., Bettaibi, N.: Wavelet transforms in quantum calculus. J. Nonlinear Math. Phys. 13(3), 492-506 (2006)

8. Fitouhi, A., Bettaibi, N.: Applications of the Mellin transform in quantum calculus. J. Math. Anal. Appl. 328, 518-534 (2007)

9. Salem, A., Ucar, F.: The $q$-analogue of the $E_{-}\{2 ; 1\}$-transform and its applications. Turk. J. Math. 40(1), 98-107 (2016)

10. Al-Omari, S.K.Q.: On $q$-analogues of the natural transform of certain $q$-Bessel functions and some application. Filomat 31(9), 2587-2598 (2017)

11. Al-Omari, S.K.Q., Baleanu, D., Purohit, S.D.: Some results for Laplace-type integral operator in quantum calculus. Adv. Differ. Equ. 2018, 124 (2018)

12. Uçar, F.: q-Sumudu transforms of q-analogues of Bessel functions. Sci. World J. 2014, Article ID 327019 (2014)

13. Al-Omari, S.K.Q.: On $q$-analogues of the Mangontarum transform for certain $q$-Bessel functions and some application. J. King Saud Univ., Sci. 28(4), 375-379 (2016)

14. Hahn, W.: Beitrage zur theorie der heineschen reihen, die 24 integrale der hypergeometrischen q-differenzengleichung, das q-analog on der Laplace transformation. Math. Nachr. 2, 340-379 (1949)

15. Al-Omari, S.K.Q: Certain results related to the $\mathrm{N}$-transform of a certain class of functions and differential operators. Adv. Differ. Equ. 2018, 7 (2018)

16. Kac, V.G., Cheung, P.: Quantum Calculus. Universitext. Springer, New York (2002)

17. Exton, H.: q-Hypergeometric Functions and Applications. Halsted, New York (1983)

18. Goldman, R., Simeonov, P., Simsek, Y.: Generating functions for the q-Bernstein bases. SIAM J. Discrete Math. 28(3), 1009-1025 (2014)

19. Yurekli, O., Sadek, I.: A Parseval-Goldstein type theorem on the Widder potential transform and its applications. Int. J. Math. Math. Sci. 14(3), 517-524 (1991)

20. Al-Omari, S.K.Q.: An extension of certain integral transform to a space of Boehmians. J. Assoc. Arab Univ. Basic Appl. Sci. 17, 36-42 (2015)

\section{Submit your manuscript to a SpringerOpen ${ }^{\circ}$ journal and benefit from:}

- Convenient online submission

- Rigorous peer review

- Open access: articles freely available online

- High visibility within the field

- Retaining the copyright to your article

Submit your next manuscript at $\boldsymbol{~ s p r i n g e r o p e n . c o m ~}$ 